\title{
A Call to Action: Moving the Scientific Publication Process toward Social Justice
}

Susan J. Cheng ${ }^{1,2,3^{*}}$, Maryam Zaringhalam ${ }^{1}$, Ana Paula S. Carvalho ${ }^{1,4,5}$, Rebecca Barnes ${ }^{1,6}$, Gretchen Goldman $^{1,7}$, Juniper Simonis ${ }^{1,8}$, Rocío P. Caballero-Gill ${ }^{9,10,11}$, Melissa S. Creary ${ }^{1,12}$, Adriana Guatame-Garcia ${ }^{9,13}$, Ana Maria Porras ${ }^{14,15}$, Alejandra Sanchez-Rios ${ }^{9,16}$, Stefanie C. Bodison $^{1,17}$, Wendy Bohon ${ }^{1,18}$, Promita Chakraborty ${ }^{19}$, Lauren Edwards ${ }^{1}$, Seanna Leath ${ }^{1,20}$, Alison R. Marklein ${ }^{1,21}$, Nicole A. Williams ${ }^{1,22}$, Kelly S. Ramirez ${ }^{1,23}$, Jane Zelikova ${ }^{1,24}$

*Correspondence: Chengs@umich.Edu

${ }^{1} 500$ Women Scientists

${ }^{2}$ Department of Ecology and Evolutionary Biology, University of Michigan, Ann Arbor, MI, USA

${ }^{3}$ Center for Research on Learning and Teaching, University of Michigan, Ann Arbor, MI, USA

${ }^{4}$ Department of Entomology and Nematology, University of Florida, Gainesville, FL, USA

${ }^{5}$ McGuire Center for Lepidoptera and Biodiversity, Florida Museum of Natural History, University of Florida, Gainesville, FL, USA

${ }^{6}$ Environmental Studies Program, Colorado College, Colorado Springs, CO, USA

${ }^{7}$ Union of Concerned Scientists, Washington, DC, USA

${ }^{8}$ DAPPER Stats, Portland, OR, USA

${ }^{9}$ GeoLatinas - Latinas in Earth and Planetary Sciences

${ }^{10}$ Mazak LLC., Puerto Rico, USA

${ }^{11}$ Department of Earth, Environmental and Planetary Sciences, Brown University, Providence, RI, USA

${ }^{12}$ School of Public Health, Department of Health Management and Policy, University of Michigan, Ann Arbor, MI, USA

${ }^{13}$ Department of Geoscience and Engineering, Delft University of Technology, the Netherlands

${ }^{14}$ Meinig School of Biomedical Engineering, Cornell University, Ithaca, NY, USA

${ }^{15}$ LatinXinBME

${ }^{16}$ Oceanography at National Taiwan University, Taipei City

${ }^{17}$ Chan Division of Occupational Science \& Occupational Therapy, University of Southern California, Los Angeles, CA, USA

${ }^{18}$ Incorporated Research Institutions for Seismology, Washington, DC, USA

${ }^{19}$ QuezyLab, California, USA

${ }^{20}$ Department of Psychology, University of Virginia, Charlottesville, VA, USA

${ }^{21}$ Department of Environmental Sciences, University of California, Riverdale, CA, USA

${ }^{22}$ The Harriet W. Sheridan Center for Teaching and Learning, Brown University, Providence, RI, USA

${ }^{23}$ Department of Biological Sciences, University of Texas at EI Paso, TX, USA

${ }^{24}$ University of Wyoming, Department of Botany, Laramie, WY, USA 


\begin{abstract}
Many scientific journals have committed to advancing diversity, equity, and inclusion, but publish articles counter to this goal. We propose actions the scientific community should take to move research and the publication process toward more rigorous and socially-just standards.
\end{abstract}

\title{
Introduction
}

Science is never neutral. It has a long history of upholding racism, misogyny, ableism, colonialism, and other harmful systems of power. ${ }^{1}$ Even today, high-profile journals publish articles that perpetuate outdated essentialist definitions of binary sex and gender ${ }^{2}$, try to link genetics to income and educational attainment, and attempt to measure "trustworthiness" with facial features. Last year, Nature Communications published a study that counters Nature Research's commitments to diversity, equity, and inclusion (DEI). ${ }^{3}$ Journal decisions to publish articles like the now-retracted AIShebli et al. 2020-which suggests that women scientists are less impactful mentors and mentees-are evidence of larger systemic failures and the enduring power of systems of oppression in science.

Many of the critiques of AlShebli et al. 2020 have already been outlined by critics and peer reviewers, spurring an investigation and subsequent retraction. Here, we turn our attention to how the scientific community can begin to critically re-evaluate scientific norms using lessons and frameworks from other disciplines, such as those in Ford and Airhihenbuwa 2010, Boyd et al. 2020, and Gillborn et al. 2018. ${ }^{4-6}$ Such critical reflections are necessary if we are to have a scientific publication process-from research design to print-aligned with the DEI and justice (DEIJ) commitments we profess.

\section{How Can We Make the Scientific Publication Process More Rigorous and Socially Just?}

Critically examine and challenge existing scholarship. To conduct rigorous and socially just research, we must meaningfully engage with existing scholarship, including in adjacent disciplines that inform the broader context of our studies. We also need to be critical of scholarship-challenging how past studies and frameworks may perpetuate stereotypes or harmful power hierarchies. ${ }^{4}$ Studies on gender and/or mentorship, for example, require scientists to bring in scholarship on gender biases in academia, gender identity misconceptions, 
and effective mentorship practices. Engaging critically with these areas of scholarship teaches us lessons that were not applied in AIShebli et al. 2020: that (a) sex is distinct from gender and (b) neither sex nor gender are binary. Furthermore, that there exists a published body of literature using name-to-gender inference tools does not mean that we should continue using them. We know inferring gender from name can violently misgender people and erase nonbinary, transgender, and gender non-conforming individuals. ${ }^{7}$ We also know inferring gender from name can exclude populations if criteria do not account for different social contexts, including variations in culture and language. ${ }^{8}$ Continuing to learn from and identify ways that scholarship perpetuates harm is critical for using science research to advance social justice.

\section{Recognize that research processes always already reflect society's power dynamics.} "Culture and power shape knowledge production by establishing the processes by which understandings are generated and disseminated." To visibilize how culture and power are part of the scientific process, researchers need to identify and challenge how power dynamics infiltrate study design, constructs, methodologies, and interpretation. In academia, current systems of power emphasize publications and funding as measures of success. Rather than challenge this norm, AIShebli et al. reinforced it, using the number of publications and their citation indices as proxies for successful mentorship_-despite the well-documented problematic nature of these measures. ${ }^{9}$ Furthermore, defining impactful mentorship through co-authorship unnecessarily commodifies the mentor-mentee relationship and ignores that: (1) co-authorship does not imply mentorship and (2) effective mentors do far more than help mentees publish papers. Effective mentors may also boost morale; nominate mentees for awards or speaking opportunities; and provide strategies for navigating a system not created for women, non-binary individuals, and "persons excluded because of their ethnicity or race" (PEERs). ${ }^{10,11}$ But these activities, which are disproportionately performed by historically-excluded and marginalized researchers, were not assessed by AIShebli et al. 2020 ${ }^{12,13}$ The lack of qualitative assessment of these relationships removes the human connection critical for successful mentorship while also reinforcing problematic academic norms.

Acknowledge that data are not neutral. Researchers have the ethical responsibility to identify how data may "embody the dominant...assumptions that shape contemporary society" because "all data [are] manufactured and all analysis is driven by human decisions." AIShebli et al. 2020 did not account for how their dataset spanning "over a century of research" was biased and reflected the systemic barriers that women scientists have faced. ${ }^{3}$ Throughout the 20th century, 
women scientists were given fewer resources and opportunities, resulting in under-valued and under-reported accomplishments. ${ }^{14}$ Marginalization has been even worse for PEERs, whose participation and contributions have been further suppressed by white supremacy and colonialism; yet discussion of intersectionality was absent from AIShebli et al. 2020. ${ }^{15}$ Rather than challenge and correct for these historical priors, these social inequities were normalized and reinforced through the authors' work. Authors, editors, and reviewers are responsible for appropriately framing data, including by identifying how social inequities and power dynamics manifest themselves in analyses and findings. ${ }^{5,6}$

Think critically about broader impacts and potential harms. Research that advances social justice requires "researchers use the knowledge obtained through their studies to help disrupt one or more causes of the inequities." AIShebli et al. 2020 conclude that women-women mentor-mentee pairs are less productive, perpetuating sexist ideas that women have less value than men. This interpretation relocates the blame to women, rather than the unjust systems that propagate unequal access to resources and produce inhospitable working environments. ${ }^{16}$ This interpretation could lead mentors to prioritize working with students who are men, further reducing opportunities for other genders in science. While the authors noted that several societal factors were "out of the scope of current study," 3 these factors are critical for correctly framing their research questions and conclusions, and must be used to set the study's scope and discussion of results.

Account for biases in the publication process. Journal review processes must account for the biases baked into science by re-evaluating how reviewers and editors decide what to publish (or not) and why. ${ }^{5}$ For example, how do we ensure reviewers assess potential biases of a study? Do standards exist to ensure reviewer comments are addressed adequately? As more studies address DEIJ in mentoring, education, collaborations, and research in general, we need to value expertise in those areas. Doing so means adding experts in education, critical theories (e.g., critical race theory, decolonial studies, disability studies, queer theory, gender studies), and science, technology, and society studies to the reviewer pool. We also need to acknowledge when journals and the peer-review process fail-and adjust the process to address those failures. While the paper has been retracted, we must continue to hold the scientific community_especially scientific journals_accountable for research that is poorly conducted. 


\section{Moving Forward}

Breaking the pattern of publishing faulty and harmful research requires looking back through the history of science, grappling with its many harms, and using that evidence to build better, more equitable and just scientific norms. We must remember that "[s]ocial injustices...are not located exclusively in the large-scale institutional background, but are found also in individual behaviors and relationships that promote inequality." 17 Beyond individual action, we need to collectively hold scientific institutions and journals accountable when they fail to lead with and uphold our values. There are several actionable things we can do today:

- Examine and acknowledge positionality. Positionality (or reflexivity) statements are one way researchers can explicitly acknowledge and discuss their biases and how they shape findings. ${ }^{6}$ These kinds of self-reflections could also be done by editors, reviewers, and organizations/institutions.

- Foreground methods. Methods form the foundation of the conclusions we draw. The "Materials and Methods" section should always be part of the main text, preferably before the results, so readers can readily contextualize and evaluate findings and interpretations.

- Define constructs. Researchers must sufficiently define and provide supporting references for the constructs that are central to their work, including the underlying assumptions within the theoretical constructs used in their scholarship.

- Ensure peer reviewers have appropriate expertise. Scientific journals need peer reviewers who have expertise in how society intersects with and informs knowledge production. Editors and reviewers should also represent diverse lived experiences and identities, such as gender, sexuality, race, ethnicity, disability, and nationality.

- Honor and incorporate critical commentary. Peer review does not stop when the paper is published. Scientists cannot ignore when studies are published despite their lack of rigor-we have a responsibility to call attention to their flaws. When this happens, journals should address community concerns, such as adding a disclaimer to the top of the publication and references to pieces that flag limitations and critical considerations of the study.

- Acknowledge science's long and violent history of exclusion. For journals-and the scientific community at large —-to fulfill their commitments to DEIJ, we must all do our part to interrogate the histories of our fields and acknowledge the harm they have caused. 
We must move beyond the incomplete, incorrect, and narrow view that blames historically excluded people for the barriers and limitations they face in science. As scientists, reviewers, editors, and leaders of scientific institutions, we must commit to doing better if we are "not only to understand inequities, but to eliminate them."4

\section{References}

1. Harding, S. G. The 'Racial' economy of science : toward a democratic future. (Indiana University Press, 1993).

2. Richie, C. Sex, not gender. A plea for accuracy. Exp. Mol. Med. 51, 1-1 (2019).

3. AIShebli, B., Makovi, K. \& Rahwan, T. The association between early career informal mentorship in academic collaborations and junior author performance. Nat. Commun. 11, 5855 (2020).

4. Ford, C. L. \& Airhihenbuwa, C. O. The public health critical race methodology: Praxis for antiracism research. Soc. Sci. Med. 71, 1390-1398 (2010).

5. Boyd, R. W., Lindo, E. G., Weeks, L. D. \& McLemore, M. R. On Racism: A New Standard For Publishing On Racial Health Inequities | Health Affairs Blog. Health Aff. (2020) doi:10.1377/hblog20200630.939347.

6. Gillborn, D., Warmington, P. \& Demack, S. QuantCrit: education, policy, 'Big Data' and principles for a critical race theory of statistics. Race Ethn. Educ. 21, 158-179 (2018).

7. Simonis, J. L. End the Gender Binary. (Zenodo, 2019). doi:10.5281/zenodo.3526299.

8. Mihaljević, H., Tullney, M., Santamaría, L. \& Steinfeldt, C. Reflections on Gender Analyses of Bibliographic Corpora. Front. Big Data 2, (2019).

9. Egghe, L. The Hirsch index and related impact measures. Annu. Rev. Inf. Sci. Technol. 44, 65-114 (2010).

10. National Academies of Sciences, Engineering, and Medicine. The Science of Effective Mentorship in STEMM. (National Academies Press, 2019). doi:10.17226/25568.

11. Asai, D. J. Race Matters. Cell 181, 754-757 (2020).

12. Guarino, C. M. \& Borden, V. M. H. Faculty Service Loads and Gender: Are Women Taking Care of the Academic Family? Res. High. Educ. 58, 672-694 (2017).

13. Cleveland, D. R., Sailes, D. J., Gilliam, D. E. \& Watts, J. A Theoretical Focus on Cultural Taxation: Who Pays for It in Higher Education. Adv. Soc. Sci. Res. J. 5, (2018).

14. Kohlstedt, S. G. Sustaining Gains: Reflections on Women in Science and Technology in 20th-Century United States. NWSA J. 16, 1-26 (2004).

15. Schell, C. J. et al. Recreating Wakanda by promoting Black excellence in ecology and evolution. Nat. Ecol. Evol. 4, 1285-1287 (2020).

16. National Academies of Sciences, Engineering, and Medicine. Sexual Harassment of Women: Climate, Culture, and Consequences in Academic Sciences, Engineering, and Medicine. (The National Academies Press, 2018). doi:10.17226/24994.

17. Hammond, J. W. Toward a Social Justice Historiography for Writing Assessment. in Writing Assessment, Social Justice, and the Advancement of Opportunity 41-70 (University Press of Colorado, 2018). 